\title{
SCHUR MULTIPLIERS OF SPECIAL $p$-GROUPS OF RANK 2
}

\author{
SUMANA HATUI
}

\begin{abstract}
Let $G$ be a special $p$-group with center of order $p^{2}$. Berkovich and Janko asked to find the Schur multiplier of $G$ in [1 Problem 2027]. In this article we answer this question by explicitly computing the Schur multiplier of these groups.
\end{abstract}

\section{INTRODUCTION}

Let $G$ be a finite $p$-group and $d(G)$ denotes the cardinality of minimal generating set of $G$. The commutator subgroup and center of $G$ are denoted by $G^{\prime}$ and $\mathrm{Z}(G)$ respectively. By $E S_{p^{k}}\left(p^{n}\right)$, we denote the extraspecial $p$-group of order $p^{n}$ of exponent $p^{k}, k=1,2$ and by $\mathbb{Z}_{p}^{(k)}$, we denote the elementary abelian $p$-group of rank $k$ for $k \geq 1$. For a prime $p, G^{p}$ denotes the group generated by the set $\left\{g^{p} \mid g \in G\right\}$. A finite $p$-group $G$ is called special p-group of rank $k$ if $G^{\prime}=\mathrm{Z}(G)$ is an elementary abelian $p$-group of order $p^{k}$ and $G / G^{\prime}$ is elementary abelian. A group $G$ is called capable group if there exists a group $H$ such that $G \cong H / \mathrm{Z}(H)$. The epicenter of a group $G$ is denoted by $Z^{*}(G)$, which is the smallest central subgroup $K$ of $G$ such that $G / K$ is capable.

The Schur multiplier of a group $G$, denoted by $\mathrm{M}(G)$, is the second integral homology group $\mathrm{H}_{2}(G, \mathbb{Z})$ which was introduced by Schur in 1904 in his fundamental work on projective representation of groups. There has been a great importance in understanding the Schur multipliers of finite $p$-groups in recent past. Here we are interested to compute Schur multiplier of special $p$-groups of rank 2 . The special $p$-groups of minimum rank are the extraspecial $p$-groups and their Schur multiplier was studied in [3. The Schur multiplier of special $p$-groups having maximum rank was studied in [12. In this article we determine the Schur multiplier of special $p$-groups of rank 2 and that answered the question which was asked by Berkovich and Janko in [1, Problem 2027].

Recall that, there are two extraspecial $p$-groups of order 8 upto isomorphism, both are of exponent 4 , one is quaternian group $Q_{8}$ which has trivial Schur multiplier and another is dihedral group $D_{8}$ which has Schur multiplier of order 2 (see [8, Theorem 3.3.6]).

We state our main results now. The following result describes the Schur multiplier of $G$ when $G^{p}=G^{\prime} \cong \mathbb{Z}_{p} \times \mathbb{Z}_{p}$.

Theorem 1.1. Let $G$ be a special p-group of rank 2 with $d=d(G)$ and $G^{p}=G^{\prime}$. Then the following assertions hold:

(a) Either $Z^{*}(G)=\mathrm{Z}(G)$ or $G$ is capable.

(b) $\mathrm{M}(G)$ is elementary abelian of order $p^{\frac{1}{2} d(d-1)-2}$ if and only if $Z^{*}(G)=\mathrm{Z}(G)$.

(c) $\mathrm{M}(G)$ is of order $p^{\frac{1}{2} d(d-1)-1}$ of exponent at most $p^{2}$ if and only if $G$ is capable.

(d) For every central subgroup $Z$ of order $p, G / Z$ is isomorphic to

$$
E S_{p^{2}}\left(p^{2 m+1}\right) \times \mathbb{Z}_{p}^{(d-2 m)}(m \geq 2), E S_{p^{2}}\left(p^{3}\right) \times \mathbb{Z}_{p}^{(d-2)} \text { or } Q_{8} \times \mathbb{Z}_{2}^{(d-2)} .
$$

By [5, Proposition 3] and Theorem 1.1] we have the immediate corollary.

Corollary 1.2. If $G$ is a special p-group of rank 2 of order $p^{n}(n \geq 8)$ with $G^{p}=G^{\prime}$, then $\mathrm{M}(G)$ is elementary abelian of order $p^{\frac{1}{2}(n-2)(n-3)-2}$.

2010 Mathematics Subject Classification. 20J99, 20 D15.

Key words and phrases. Schur Multiplier, Finite $p$-group. 
The following result describes the Schur multiplier of $G$ when $G^{p}$ is cyclic of prime order.

Theorem 1.3. If $G$ is a special p-group of rank 2 with $d=d(G)$ and $G^{p} \cong \mathbb{Z}_{p}$, then the following assertions hold:

(a) $G$ is not capable and either $Z^{*}(G)=\mathrm{Z}(G)$ or $Z^{*}(G)=G^{p}$.

(b) $\mathrm{M}(G)$ is elementary abelian.

(c) The following are equivalent:

(i) $\mathrm{M}(G)$ is of order $p^{\frac{1}{2} d(d-1)-2}$.

(ii) $Z^{*}(G)=\mathrm{Z}(G)$.

(iii) $G / G^{p} \cong E S_{p}\left(p^{2 m+1}\right) \times \mathbb{Z}_{p}^{(d-2 m)}, m \geq 2$.

(d) The following are equivalent:

(i) $\mathrm{M}(G)$ is of order $p^{\frac{1}{2} d(d-1)}$.

(ii) $Z^{*}(G)=G^{p}$.

(iii) $G / G^{p} \cong E S_{p}\left(p^{3}\right) \times \mathbb{Z}_{p}^{(d-2)}$.

Now we are left with only one case when $G^{p}$ is trivial and in this case the Schur multiplier of $G$ is studied in the following result.

Theorem 1.4. If $G$ is a special p-group of rank 2 with $d=d(G)$ and $G^{p}=1$, $p$ odd, then the following assertions hold:

(a) $\mathrm{M}(G)$ is elementary abelian.

(b) $p^{\frac{1}{2} d(d-1)-2} \leq|\mathrm{M}(G)| \leq p^{\frac{1}{2} d(d-1)+3}$.

(c) $G$ is capable if and only if $G$ is isomorphic to one of the following groups:

(i) $\Phi_{4}\left(1^{5}\right)=\left\langle\alpha, \alpha_{1}, \alpha_{2}, \beta_{1}, \beta_{2} \mid\left[\alpha_{i}, \alpha\right]=\beta_{i}, \alpha^{p}=\alpha_{i}^{p}=\beta_{i}^{p}=1(i=1,2)\right\rangle$.

(ii) $\Phi_{12}\left(1^{6}\right)=E S_{p}\left(p^{3}\right) \times E S_{p}\left(p^{3}\right)$,

(iii) $\Phi_{13}\left(1^{6}\right)=\left\langle\alpha_{1}, \alpha_{2}, \alpha_{3}, \alpha_{4}, \beta_{1}, \beta_{2}\right|\left[\alpha_{i}, \alpha_{i+1}\right]=\beta_{i},\left[\alpha_{2}, \alpha_{4}\right]=\beta_{2}, \alpha_{i}^{p}=\alpha_{3}^{p}=\alpha_{4}^{p}=$ $\left.\beta_{i}^{p}=1(i=1,2)\right\rangle$,

(iv) $\Phi_{15}\left(1^{6}\right)=\left\langle\alpha_{1}, \alpha_{2}, \alpha_{3}, \alpha_{4}, \beta_{1}, \beta_{2}\right|\left[\alpha_{i}, \alpha_{i+1}\right]=\beta_{i},\left[\alpha_{3}, \alpha_{4}\right]=\beta_{1},\left[\alpha_{2}, \alpha_{4}\right]=$ $\left.\beta_{2}^{g}, \alpha_{i}^{p}=\alpha_{3}^{p}=\alpha_{4}^{p}=\beta_{i}^{p}=1(i=1,2)\right\rangle$, where $g$ is non-quadratic residue modulo $p$.

(v) $T=\left\langle x_{1}, \cdots, x_{5}, c_{1}, c_{2}\right|\left[x_{2}, x_{1}\right]=\left[x_{5}, x_{3}\right]=c_{1},\left[x_{3}, x_{1}\right]=\left[x_{5}, x_{4}\right]=c_{2}, x_{i}^{p}=$ $\left.c_{j}^{p}=1,1 \leq i \leq 5,1 \leq j \leq 2\right\rangle$.

(d) $|\mathrm{M}(G)|=p^{\frac{1}{2} d(d-1)+3}$ if and only if $G$ is isomorphic to $\Phi_{4}\left(1^{5}\right)$.

(e) $|\mathrm{M}(G)|=p^{\frac{1}{2} d(d-1)+2}$ if and only if $G$ is isomorphic to $\Phi_{12}\left(1^{6}\right), \Phi_{13}\left(1^{6}\right)$, or $\Phi_{15}\left(1^{6}\right)$.

$(f)|\mathrm{M}(G)|=p^{\frac{1}{2} d(d-1)-1}$ if and only if $G$ is isomorphic to $T$.

(g) $\mathrm{M}(G)$ is of order $p^{\frac{1}{2} d(d-1)-2}$ if and only if $Z^{*}(G)=\mathrm{Z}(G)$. In this case, $G / Z \cong$ $E S_{p}\left(p^{2 m+1}\right) \times \mathbb{Z}_{p}^{(d-2 m)}, m \geq 2$, for every central subgroup $Z$ of order $p$.

(h) $\mathrm{M}(G)$ is of order $p^{\frac{1}{2} d(d-1)}$ if and only if $Z^{*}(G) \cong \mathbb{Z}_{p}$. In this case, $G / Z^{*}(G) \cong$ $E S_{p}\left(p^{3}\right) \times \mathbb{Z}_{p}^{(d-2)}$.

The following result is for $p=2$.

Theorem 1.5. Let $G$ be a special 2-group of rank 2. Then $G^{2}=G^{\prime}$ holds.

\section{Preliminaries}

For a finite group $G$ of class 2 with $G / G^{\prime}$ elementary abelian, the following construction is given in [3]. We consider $G / G^{\prime}$ and $G^{\prime}$ as vector spaces over $\mathbb{F}_{p}$, which we denote by $V, W$ respectively. The bilinear map $(-,-): V \times V \rightarrow W$ is defined by

$$
\left(v_{1}, v_{2}\right)=\left[g_{1}, g_{2}\right]
$$

for $v_{1}, v_{2} \in V$ such that $v_{i}=g_{i} G^{\prime}, i \in\{1,2\}$ for some $g_{1}, g_{2} \in G$. Let $X_{1}$ be the subspace of $V \otimes W$ spanned by all

$$
v_{1} \otimes\left(v_{2}, v_{3}\right)+v_{2} \otimes\left(v_{3}, v_{1}\right)+v_{3} \otimes\left(v_{1}, v_{2}\right)
$$


for $v_{1}, v_{2}, v_{3} \in V$. Consider a map $f: V \rightarrow W$ given by

$$
f\left(g G^{\prime}\right)=g^{p}
$$

for $g \in G$. Let $X_{2}$ be the subspace spanned by all $v \otimes f(v), v \in V$, and take

$$
X:=X_{1}+X_{2} \text {. }
$$

Now consider a homomorpism $\sigma: V \wedge V \rightarrow(V \otimes W) / X$ given by

$$
\sigma\left(v_{1} \wedge v_{2}\right)=\left(v_{1} \otimes f\left(v_{2}\right)+\left(\begin{array}{l}
p \\
2
\end{array}\right) v_{2} \otimes\left(v_{1}, v_{2}\right)\right)+X .
$$

Then there exists an abelian group $M^{*}$ with a subgroup $N$ isomorphic to $(V \otimes W) / X$, such that

$$
1 \rightarrow N \rightarrow M^{*} \stackrel{\xi}{\rightarrow} V \wedge V \rightarrow 1
$$

is exact and

$$
\sigma \xi(\alpha)=\alpha^{p} \text { for } \alpha \in M^{*}
$$

Now we consider a homomorphism $\rho: V \wedge V \rightarrow W$ given by

$$
\rho\left(v_{1} \wedge v_{2}\right)=\left(v_{1}, v_{2}\right)
$$

for all $v_{1}, v_{2} \in V$. Notice that $\rho$ is an epimorphism. We let $M$ be the subgroup of $M^{*}$ containing $N$ such that $M / N \cong \operatorname{Ker} \rho$. We use this notation throughout the paper without further reference.

With the above setting, we have

Theorem 2.1. ([3, Theorem 3.1]) $\mathrm{M}(G) \cong M$.

Note: It is easy to observe that $X_{1}$ is generated by the set

$$
\left\{\bar{x}_{1} \otimes\left[x_{2}, x_{3}\right]+\bar{x}_{2} \otimes\left[x_{3}, x_{1}\right]+\bar{x}_{3} \otimes\left[x_{1} . x_{2}\right] \mid x_{1}, x_{2}, x_{3} \in S\right\},
$$

where $S$ is a set of generators of $G$ and $\bar{x}$ is the image of $x$ in $G / G^{\prime}$.

Suppose $G$ has a free presentation $F / R$. Let $Z=S / R$ be a central subgroup of $G$. Then the map from $\left(F / F^{\prime} R\right) \times(S / R)$ to $\left(F^{\prime} \cap R\right) /[F, R]$ defined by $\left(x F^{\prime} R, s R\right) \mapsto[x, s][F, R]$ is a well-defined bilinear map and induces a homomorphism $\lambda_{Z}:\left(G / G^{\prime}\right) \otimes Z \rightarrow \mathrm{M}(G)$, called the Ganea map.

Theorem 2.2 (4]). Let $Z$ be a central subgroup of a finite group $G$. Then the following sequence is exact

$$
\left(G / G^{\prime}\right) \otimes Z \stackrel{\lambda_{Z}}{\rightarrow} \mathrm{M}(G) \stackrel{\mu}{\rightarrow} \mathrm{M}(G / Z) \rightarrow G^{\prime} \cap Z \rightarrow 1
$$

Theorem $2.3([2])$. Let $Z$ be a central subgroup of a finite group $G$. Then $Z \subseteq Z^{*}(G)$ if and only if $\left(G / G^{\prime}\right) \otimes Z=\operatorname{Ker} \lambda_{Z}$.

By [8, Corollary 3.2.4], we have $X=\operatorname{Ker} \lambda_{\mathrm{Z}(G)}$. Hence by Theorem 2.3, we have the following result:

Lemma 2.4. Let $Z$ be a central subgroup of a group $G$ of nilpotency class 2 . Then $Z \subseteq$ $Z^{*}(G)$ if and only if $\left(G / G^{\prime}\right) \otimes Z$ is contained in $X$.

Let $v_{1}, v_{2}, \ldots, v_{d}$ be the generators of $V$ such that $\left\{f\left(v_{1}\right), f\left(v_{2}\right), \ldots, f\left(v_{r}\right)\right\}$ is a basis of $G^{p}$. Then the set

$$
\left\{v_{i} \otimes f\left(v_{i}\right), v_{l} \otimes f\left(v_{i}\right),\left(v_{i} \otimes f\left(v_{j}\right)+v_{j} \otimes f\left(v_{i}\right)\right) \mid 1 \leq i<j \leq r,(r+1) \leq l \leq d\right\}
$$

forms a basis of $X_{2}$, from which the following result follows.

Proposition 2.5 (Proposition 3.3 of [12]). Let $G$ be a special p-group with $d=d(G)$ and $G^{p}$ of order $r$. Then $\left|X_{2}\right|=p^{r d-\frac{1}{2} r(r-1)}$. 
By Theorem 2.2, we have

$$
\frac{|\mathrm{M}(G)|}{\left|\operatorname{Im} \lambda_{Z}\right|}=\frac{|\mathrm{M}(G / Z)|}{\left|G^{\prime} \cap Z\right|} .
$$

As $X=\operatorname{Ker} \lambda_{\mathrm{Z}(G)}$, so $\left|\operatorname{Im} \lambda_{\mathrm{Z}(G)}\right|=\frac{p^{2 d}}{|X|}$. Hence, by (2.1), taking $Z=\mathrm{Z}(G)$, we have

$$
|\mathrm{M}(G)|=p^{\frac{1}{2} d(d-1)-2} \cdot \frac{p^{2 d}}{|X|}
$$

Now we recall the following results which will be used in the proof of the main results.

Theorem 2.6 (Main Theorem of [9]). Let $G$ be a p-group of order $p^{n}$. Then $|\mathrm{M}(G)|=$ $p^{\frac{1}{2}(n-1)(n-2)+1}$ if and only if $G \cong E S_{p}\left(p^{3}\right) \times \mathbb{Z}_{p}^{(n-3)}$.

Theorem 2.7 (Theorem 21 of [10]). Let $G$ be a p-group of order $p^{n}$. Then $|\mathrm{M}(G)|=$ $p^{\frac{1}{2}(n-1)(n-2)}$ if and only if $G$ is isomorphic to one of the following groups.

(i) $G \cong \mathbb{Z}_{p^{2}} \times \mathbb{Z}_{p}^{(n-2)}$,

(ii) $G \cong D_{8} \times \mathbb{Z}_{2}^{(n-3)}$,

(iii) $G \cong \mathbb{Z}_{p}^{(4)} \rtimes \mathbb{Z}_{p}(p \neq 2)$.

Theorem 2.8 (Theorem 11 of [11]). Let $G$ be a group of order $p^{n}$ such that $G / G^{\prime}$ is elementary abelian of order $p^{n-1}$. Then $|\mathrm{M}(G)|=p^{\frac{1}{2}(n-1)(n-2)-1}$ if and only if $G$ is isomorphic to one of the following groups.

(i) $E S_{p^{2}}\left(p^{3}\right) \times \mathbb{Z}_{p}^{(n-3)}$,

(ii) $Q_{8} \times \mathbb{Z}_{2}^{(n-3)}$,

(iii) $E S\left(p^{2 m+1}\right) \times \mathbb{Z}_{p}^{(n-2 m-1)}(m \geq 2)$.

\section{Proofs}

In this section we prove our main results.

Proof of Theorem 1.1: Consider $G^{p}=G^{\prime} \cong \mathbb{Z}_{p} \times \mathbb{Z}_{p}$. Now by Proposition 2.5, $\left|X_{2}\right|=$ $p^{2 d-1}$. Let $v_{1}, v_{2} \in V$ such that $\left\{f\left(v_{1}\right), f\left(v_{2}\right)\right\}$ is a basis of $W$. Observe that $X_{2}$ is generated by the set

$$
\left\{v_{i} \otimes f\left(v_{i}\right), v_{j} \otimes f\left(v_{i}\right),\left(v_{1} \otimes f\left(v_{2}\right)+v_{2} \otimes f\left(v_{1}\right)\right) \mid i=1,2 \text { and } 3 \leq j \leq d\right\} .
$$

Hence $p^{2 d} \geq|X| \geq p^{2 d-1}$ and by (2.2),

$$
p^{\frac{1}{2} d(d-1)-2} \leq|\mathrm{M}(G)| \leq p^{\frac{1}{2} d(d-1)-1} .
$$

(a) Observe that $|X|=p^{2 d}$, i.e., the set $\left\{v_{1} \otimes f\left(v_{2}\right), v_{2} \otimes f\left(v_{1}\right)\right\}$ is contained in $X$ if and only if $Z^{*}(G)=\mathrm{Z}(G)$, follows by Lemma 2.4. Another possibility is $|X|=p^{2 d-1}$, i.e., $X_{1} \subseteq X_{2}$ if and only if $v_{1} \otimes f\left(v_{2}\right), v_{2} \otimes f\left(v_{1}\right)$ are not in $X$. Hence, by Lemma 2.4 $Z^{*}(G)=1$. So $G$ is capable.

(b) By (2.2),$|X|=p^{2 d}$ if and only if

$$
|\mathrm{M}(G)|=p^{\frac{1}{2} d(d-1)-2}
$$

By Theorem 2.3 and Theorem 2.2, taking $Z=\mathrm{Z}(G)$, we see that $\mathrm{M}(G)$ embeds in $\mathrm{M}(G / \mathrm{Z}(G))$ which is elementary abelian. So in this case $\mathrm{M}(G)$ is elementary abelian.

(c) For $x \in \mathrm{M}(G), x^{p} \in(V \otimes W) / X$ and $V \otimes W / X$ is elementary abelian, so $x^{p^{2}}=1$.

By (2.2), $X_{1} \subseteq X_{2}$ i.e., $|X|=p^{2 d-1}$ if and only if, by (2.2),

$$
|\mathrm{M}(G)|=p^{\frac{1}{2} d(d-1)-1} \text {. }
$$

Hence, by Lemma 2.4, $Z^{*}(G)=1$. So $G$ is capable. Converse follows from $(b)$. 
(d) Observe that, in both the cases $|\mathrm{M}(G / Z)|=p^{\frac{1}{2} d(d-1)-1}$, follows from (2.1), taking $Z$ a central subgroup of order $p$. Therefore by Theorem 2.8 .

$$
G / Z \cong E S_{p^{2}}\left(p^{2 m+1}\right) \times \mathbb{Z}_{p}^{(d-2 m)},(m \geq 2), E S_{p^{2}}\left(p^{3}\right) \times \mathbb{Z}_{p}^{(d-2)} \text { or } Q_{8} \times \mathbb{Z}_{2}^{(d-2)} .
$$

Proof of Theorem 1.3: (a) Assume $G^{p}$ is cyclic of order $p$. Now by Proposition 2.5, $\left|X_{2}\right|=p^{d}$. Let $v_{1} \in V$ such that $G^{p}=\left\langle f\left(v_{1}\right)\right\rangle$. Observe that $X_{2}$ is generated by the set

$$
M^{\prime}:=\left\{v_{i} \otimes f\left(v_{1}\right), 1 \leq i \leq d\right\} .
$$

Hence by Lemma 2.4, $G^{p} \subseteq Z^{*}(G)$. Hence $G$ is not capable with $Z^{*}(G)=G^{p}$ or $Z^{*}(G)=$ $\mathrm{Z}(G)$.

Using Theorem 2.3 and taking $Z=G^{p}$ in (2.1), we have

$$
|\mathrm{M}(G)|=\frac{\left|\mathrm{M}\left(G / G^{p}\right)\right|}{p}
$$

Now let $v_{1}, v_{2} \in V$ such that $\left(v_{1}, v_{2}\right) \in G^{\prime} \backslash G^{p}$. Then The set

$$
N^{\prime}:=\left\{v_{i} \otimes\left(v_{1}, v_{2}\right)+v_{1} \otimes\left(v_{2}, v_{i}\right)+v_{2} \otimes\left(v_{i}, v_{1}\right) \mid 3 \leq i \leq d\right\}
$$

is linearly independent in $X_{1}$, so $\left|X_{1}\right| \geq p^{d-2}$. The set $M^{\prime} \cup N^{\prime}$ is linearly independent in $X$ and $M^{\prime} \cap N^{\prime}=\emptyset$. Thus

Hence by (2.2),

$$
p^{2 d} \geq|X| \geq p^{2 d-2} \text {. }
$$

$$
p^{\frac{1}{2} d(d-1)-2} \leq|\mathrm{M}(G)| \leq p^{\frac{1}{2} d(d-1)} .
$$

(c) Now similarly, as described in the proof of Theorem 1.1. we have $Z^{*}(G)=\mathrm{Z}(G)$ if and only if $|\mathrm{M}(G)|=p^{\frac{1}{2} d(d-1)-2}$ i.e., by (3.1),

$$
\left|\mathrm{M}\left(G / G^{p}\right)\right|=p^{\frac{1}{2} d(d-1)-1},
$$

which happens if and only if

$$
G / G^{p} \cong E S_{p}\left(p^{2 m+1}\right) \times \mathbb{Z}_{p}^{(d-2 m)}, m \geq 2,
$$

follows from Theorem 2.8

(d) By Theorem 2.7, it follows that there is no $G / G^{p}$ such that

$$
\left|\mathrm{M}\left(G / G^{p}\right)\right|=p^{\frac{1}{2} d(d-1)} .
$$

Thus by (3.1), $|\mathrm{M}(G)|$ cannot be of order $p^{\frac{1}{2} d(d-1)-1}$. Hence $|\mathrm{M}(G)|=p^{\frac{1}{2} d(d-1)}$ if and only if $Z^{*}(G)=G^{p}$. By (3.1),

$$
\left|\mathrm{M}\left(G / G^{p}\right)\right|=p^{\frac{1}{2} d(d-1)+1},
$$

which happens if and only if

$$
G / G^{p} \cong E S_{p}\left(p^{3}\right) \times \mathbb{Z}_{p}^{(d-2)},
$$

follows by Theorem 2.6

(b) By $(c)$ and $(d)$ it follows that $p$ must be odd. The group $G / G^{p}$ is of exponent $p$ and $p$ odd, so the homomorphism $\sigma$, described in Section 2, is trivial map and therefore, $\sigma \xi(x)=x^{p}=1$ for $x \in \mathrm{M}\left(G / G^{p}\right)$. Thus $\mathrm{M}\left(G / G^{p}\right)$ is elementary abelian. Since $G^{p} \subseteq Z^{*}(G)$, by Theorem 2.3 and Theorem $2.2, \mathrm{M}(G)$ embeds in $\mathrm{M}\left(G / G^{p}\right)$. Therefore, $\mathrm{M}(G)$ is also elementary abelian. The proof is complete now.

Proof of Theorem 1.4: (a) Since $p$ is odd and $G^{p}=1$, the homomorphism $\sigma$ described in Section 2, is the trivial map and therefore $\sigma \xi(x)=x^{p}=1$. Thus, $\operatorname{M}(G)$ is elementary abelian. 
Let $z, z^{\prime}$ be the generators of $\mathrm{Z}(G)$ and $x_{1}, x_{2}, \ldots, x_{d}$ be the generators of $G$ such that $\left[x_{1}, x_{2}\right] \in\langle z\rangle$ is non-trivial. Then the set

$$
A:=\left\{x_{i} \otimes\left[x_{1}, x_{2}\right]+x_{1} \otimes\left[x_{2}, x_{i}\right]+x_{2} \otimes\left[x_{i}, x_{1}\right] \mid 3 \leq i \leq d\right\}
$$

consists of $d-2$ linearly independent elements of $X_{1}$.

Now if for some $x_{k} \in\{3,4, \ldots, d\},\left[x_{1}, x_{k}\right] \in\left\langle z^{\prime}\right\rangle$ is non-trivial, then the set

$$
B:=\left\{x_{i} \otimes\left[x_{1}, x_{k}\right]+x_{1} \otimes\left[x_{k}, x_{i}\right]+x_{k} \otimes\left[x_{i}, x_{1}\right] \mid 3 \leq i \leq d, i \neq k\right\}
$$

consists of $d-3$ linearly independent elements of $X_{1}$. Thus, $A \cup B$ consists of $(2 d-5)$ linearly independent elements of $X_{1}$.

If $1 \neq\left[x_{2}, x_{k}\right] \in\left\langle z^{\prime}\right\rangle$, for some $k \in\{3,4, \ldots, d\}$, then a similar conclusion holds. Suppose then that $\left[x_{1}, x_{k}\right]$ and $\left[x_{2}, x_{k}\right]$ are all trivial or in $\langle z\rangle$ for all $k \in\{3,4, \ldots, d\}$. Say, $\left[x_{3}, x_{4}\right] \in$ $\left\langle z^{\prime}\right\rangle$. In this case

$$
B_{1}:=\left\{x_{i} \otimes\left[x_{3}, x_{4}\right]+x_{3} \otimes\left[x_{4}, x_{i}\right]+x_{4} \otimes\left[x_{i}, x_{3}\right] \mid 1 \leq i \leq d, i \neq 3,4\right\}
$$

consists of $d-2$ independent elements of $X_{1}$. Thus, $A \cup B_{1}$ consists of $2 d-4$ linearly independent elements of $X_{1}$.

Hence in both cases, $2 d \geq|X|=\left|X_{1}\right| \geq p^{2 d-5}$ holds. By (2.2), it follows that,

$$
p^{\frac{1}{2} d(d-1)-2} \leq|\mathrm{M}(G)| \leq p^{\frac{1}{2} d(d-1)+3},
$$

which proves $(b)$.

Now if $G$ is not capable, then by (2.1) and [9, Main Theorem] we have

$$
p^{\frac{1}{2} d(d-1)-2} \leq|\mathrm{M}(G)|=\frac{|\mathrm{M}(G / Z)|}{p} \leq p^{\frac{1}{2} d(d-1)} \text { for } Z \subseteq Z^{*}(G), Z \cong \mathbb{Z}_{p} .
$$

By Theorem 2.7 there is no $G$ and central subgroup $Z$ such that $|\mathrm{M}(G / Z)|=p^{\frac{1}{2} d(d-1)}$. Hence

$$
|\mathrm{M}(G)|=p^{\frac{1}{2} d(d-1)-2} \text { or } p^{\frac{1}{2} d(d-1)} \text {, if } G \text { is not capable. }
$$

Assume then that $G$ is capable. By [5, Proposition 3], $p^{5} \leq|G| \leq p^{7}$, with $p$ odd. If $|G|=p^{5}$, then looking through the list of groups given in [7], it follows that $G \cong \Phi_{4}\left(1^{5}\right)$. Since $|X|=p$, it follows by (2.2) that $|\mathrm{M}(G)|=p^{6}=p^{\frac{1}{2} d(d-1)+3}$.

If $|G|=p^{6}$, then looking through the list of groups given in 7 , it follows that $G \cong$ $\Phi_{12}\left(1^{6}\right), \Phi_{13}\left(1^{6}\right)$ or $\Phi_{15}\left(1^{6}\right)$. Since $|X|=p^{4}$, it follows by (2.2) that $|\mathrm{M}(G)|=p^{8}=$ $p^{\frac{1}{2} d(d-1)+2}$.

Now consider groups of order $p^{7}$ of exponent $p$. By [6], it follows that there is only one capable group

$$
\begin{array}{r}
G=\left\langle x_{1}, \ldots, x_{5}, c_{1}, c_{2}\right|\left[x_{2}, x_{1}\right]=\left[x_{5}, x_{3}\right]=c_{1},\left[x_{3}, x_{1}\right]=\left[x_{5}, x_{4}\right]=c_{2}, x_{i}^{p}=c_{j}^{p}=1, \\
1 \leq i \leq 5,1 \leq j \leq 2\rangle
\end{array}
$$

up to isomorphism. By (2.2), $|\mathrm{M}(G)|=p^{9}=p^{\frac{1}{2} d(d-1)-1}$.

Now $(c),(d),(e),(f)$ follow by (3.2).

(g) Now by (2.1) we have that $Z^{*}(G) \cong \mathrm{Z}(G)$ if and only if $|\mathrm{M}(G)|=p^{\frac{1}{2} d(d-1)-2}$, taking $Z=\mathrm{Z}(G)$. Hence by (2.1) we have that for every central subgroup $Z$ of order $p$, $|\mathrm{M}(G / Z)|=p^{\frac{1}{2} d(d-1)-1}$, thus

$$
G / Z \cong E S_{p}\left(p^{2 m+1}\right) \times \mathbb{Z}_{p}^{(d-2 m)}, m \geq 2,
$$

follows from Theorem 2.8

(h) Suppose $Z^{*}(G) \cong \mathbb{Z}_{p}$. By (3.2) and $(g)$, it follows that $|\mathrm{M}(G)|=p^{\frac{1}{2} d(d-1)}$.

Conversely suppose $|\mathrm{M}(G)|=p^{\frac{1}{2} d(d-1)}$. From the previous cases it follows that $G$ is not capable since $Z^{*}(G) \cong \mathbb{Z}_{p}$. 
In this case, we have

$$
|\mathrm{M}(G)|=\frac{\left|\mathrm{M}\left(G / Z^{*}(G)\right)\right|}{p} .
$$

By Theorem 2.6, it follows that $\left|\mathrm{M}\left(G / Z^{*}(G)\right)\right|=p^{\frac{1}{2} d(d-1)+1}$ if and only if

$$
G / Z^{*}(G) \cong E S_{p}\left(p^{3}\right) \times \mathbb{Z}_{p}^{(d-2)}
$$

The proof is complete now.

Proof of Theorem 1.5: By Theorem 1.3. it follows that there is no special 2-group of rank 2 with $G^{2} \cong \mathbb{Z}_{2}$.

Assume that $p=2$. As in the proof of Theorem 1.4, we conclude that

$$
p^{\frac{1}{2} d(d-1)-2} \leq|\mathrm{M}(G)| \leq p^{\frac{1}{2} d(d-1)+3} .
$$

Let $Z$ be a central subgroup of order 2 . If $G$ is not capable then by (2.1),

$$
2^{\frac{1}{2} d(d-1)-2} \leq|\mathrm{M}(G)|=\frac{|\mathrm{M}(G / Z)|}{2} \leq 2^{\frac{1}{2} d(d-1)} \text { for } Z \subseteq Z^{*}(G) .
$$

By Theorem 2.6, 2.7 and 2.8, there is no group $G$ and central subgroup $Z$ such that $G / Z$ of exponent 2 and $2^{\frac{1}{2} d(d-1)-1} \leq|\mathrm{M}(G / Z)| \leq 2^{\frac{1}{2} d(d-1)+1}$. Hence $G$ must be capable and $|\mathrm{M}(G / Z)| \leq 2^{\frac{1}{2} d(d-1)-2}$.

Suppose $|\mathrm{M}(G)|=2^{\frac{1}{2} d(d-1)-2+k}, 0 \leq k \leq 5$. Then by (2.2), $\left|\operatorname{Ker} \lambda_{\mathrm{Z}(G)}\right|=|X|=2^{2 d-k}$. Hence $1 \leq\left|\operatorname{Im} \lambda_{Z}\right|=2^{m} \leq 2^{k}$. By (2.1), it follows that

$$
2^{\frac{1}{2} d(d-1)-2+k-m}=\frac{|\mathrm{M}(G)|}{\left|\operatorname{Im} \lambda_{Z}\right|}=\frac{|\mathrm{M}(G / Z)|}{2} \leq 2^{\frac{1}{2} d(d-1)-3} .
$$

Hence $k-m \leq-1$, which is not possible. So there is no special 2-group of rank 2 with $G^{2}=1$. The proof is complete now.

Acknowledgement: The research of the author is partly supported by Infosys grant.

\section{REFERENCES}

[1] Y. Berkovich, Z. Janko, Groups of Prime Power Order, vol. 3, Walter de Gruyter, 2011.

[2] F. R. Beyl, U. Felgner, P. Schmid, On groups occurring as center factor groups, J. Algebra 61 (1979), 161-177.

[3] N. Blackburn, L. Evens, Schur multipliers of p-groups, J. Reine Angew. Math. 309 (1979), 100-113.

[4] T. Ganea, Homologie et extensions centrales de groupes, C. R. Acad. Sci., Paris 266 (1968), 556-568.

[5] H. Heineken, Nilpotent groups of class two that can appear as central quotient groups, Rend. Sem. Mat. Univ. Padova 84 (1990), 241-248.

[6] H. Heineken, L.C. Kappe, R.F. Morse, On the classification of special p-groups of rank two that appear as central quotient groups, Preprint.

[7] R. James, The groups of order $p^{6}$ ( $p$ an odd prime), Math. Comp. 34 (1980), 613-637.

[8] G. Karpilovsky, The Schur multiplier. London Math. Soc. Monographs, Oxford Univ. Press, 1987.

[9] P. Niroomand, On the order of Schur multiplier of non-abelian p groups, Journal of Algebra, $\mathbf{3 2 2}$ (2009), 4479-4482.

[10] P. Niroomand, A note on the Schur multiplier of groups of prime power order, Ricerche di Matematica, 61 (2012), 341-346

[11] P. Niroomand, Classifying p-groups by their Schur multipliers, Math. Rep. (Bucur.) 20(70) (2018), no. 3, 279-284.

[12] P. K. Rai, On the Schur multiplier of special p-groups, J. Pure Appl. Algebra, 222 (2018), 316-322.

Department of Mathematics, Indian Institute of Science (IISc), Bangalore-560012, India

E-mail address: sumanahatui@iisc.ac.in, sumana.iitg@gmail.com 